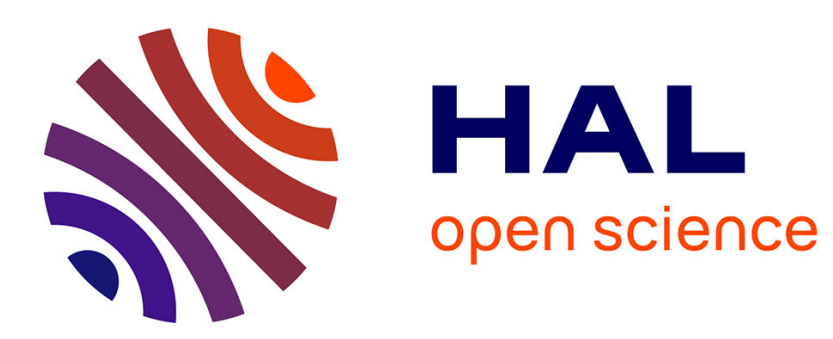

\title{
Analyse par digitalisation d'images du comportement d'affouragement d'une fourmi attine: application aux tests de choix
}

Gérard Febvay, Yvan Rahbé, Alain Kermarrec

\section{To cite this version:}

Gérard Febvay, Yvan Rahbé, Alain Kermarrec. Analyse par digitalisation d'images du comportement d'affouragement d'une fourmi attine: application aux tests de choix. Agronomie, 1986, 6 (8), pp.743749. hal-00884932

\section{HAL Id: hal-00884932 \\ https://hal.science/hal-00884932}

Submitted on 1 Jan 1986

HAL is a multi-disciplinary open access archive for the deposit and dissemination of scientific research documents, whether they are published or not. The documents may come from teaching and research institutions in France or abroad, or from public or private research centers.
L'archive ouverte pluridisciplinaire HAL, est destinée au dépôt et à la diffusion de documents scientifiques de niveau recherche, publiés ou non, émanant des établissements d'enseignement et de recherche français ou étrangers, des laboratoires publics ou privés. 


\title{
Analyse par digitalisation d'images du compor- tement d'affouragement d'une fourmi attine : application aux tests de choix
}

\author{
Gérard FEBVAY, Yvan RAHBE \& Alain KERMARREC \\ I.N.R.A., Station de Zoologie et Lutte biologique, Centre de Recherche Antilles-Guyane, F 97184 Pointe-à- \\ Pitre, Guadeloupe
}

RÉSUMÉ

\begin{abstract}
Nous décrivons l'automatisation de l'enregistrement et de l'analyse du comportement d'affouragement d'une fourmi attine Acromyrmex octospinosus (Reich) en vue de diverses applications à des tests de préférence. Le montage est réalisé à l'aide d'un micro-ordinateur couplé à une caméra électronique. Le comportement analysé est la prise de confetti (de limbe de différentes espèces végétales ou de papier filtre imbibé d'extraits à éprouver) déposés au hasard'sur une grille de choix. Les avantages et les limites de cette méthode sont discutés.
\end{abstract}

Mots clés additionnels : Hymenoptera, Formicidae, Acromyrmex octospinosus, fourmis champignonnistes, relations plantes-insectes. application to choice tests.

A new method is described for the automatic recording and analysis of foraging behaviour in the leaf-cutting ant Acromyrmex octospinosus (Reich). This method had various applications in relation to preference tests. The setup involved a micro-computer linked to a video camera. The behaviour analysed was selection of "confetti" (leaf-discs cut from various plant species or filter-paper discs treated with extracts to be tested) placed randomly on a grid. The advantages and limitations of this method are discussed.

Additional key words : Hymenoptera, Formicidae, Acromyrmex octospinosus, fungus-growing ant, leafcutting ant, plant-insect relations.

\section{INTRODUCTION}

La plupart des études éthologiques sur les insectes font appel à des tests comportementaux qui impliquent généralement la présence permanente de l'expérimentateur pour un suivi long et fastidieux. De plus, la variabilité des réponses comportementales astreint à de nombreuses répétitions. Ces expériences sont encore conduites dans la majorité des cas sous contrôle visuel et les inconvénients de cette méthode sont nombreux : diminution de l'attention de l'expérimentateur liée à la fatigue, biais involontaires dans les observations d'un expérimentateur jamais "naif " face à une expérience, difficulté de noter des comportements trop rapides... Avec le développement des techniques vidéo, certains tests éthologiques ont été automatisés par des enregistrements séquentiels d'images, par exemple lors d'un suivi de parcours d'insec- tes. Si cette méthode permet d'éliminer la plupart des inconvénients cités, elle entraîne souvent une perte d'informations directement liée à la fréquence de prise de vue. Cette perte est d'autant moins importante que la fréquence est plus élevée. En contrepartie, l'augmentation de cette dernière allonge le temps de dépouillement des résultats enregistrés, qui peut même dépasser celui de l'expérience.

La disponibilité récente de micro-ordinateurs «personnels " à des coûts aisément accessibles permet de concevoir leur utilisation pour optimiser certains tests éthologiques. Couplés à une caméra, ils permettent de conserver les avantages des méthodes vidéo (libération de l'expérimentateur, enregistrement "naïf » des comportements, ...). Leur vitesse de réponse autorise également l'étude de comportements trop rapides pour un suivi visuel. L'analyse de l'image en temps réel permet par contre de ne stocker que l'information 
significative à réutiliser. Les analyses statistiques et les exploitations graphiques des données enregistrées peuvent dès lors être aisément réalisées par des logiciels appropriés. L'expérimentateur aura ainsi à la fin d'une expérience des résultats déjà partiellement traités. Tous les tests éthologiques ne se prêtent pas à l'automatisation, cependant les avantages de l'informatique vont considérablement bouleverser ce type d'expérimentations. Déjà, quelques études font appel à ces techniques, comme l'enregistrement et l'analyse du trajet parcouru par de petits insectes (Hoy et al., 1983).

Nous décrivons l'automatisation de l'enregistrement d'un comportement d'affouragement chez la fourmi attine, Acromyrmex octospinosus (Reich), en vue d'applications à des tests de préférence végétale.

Nous privilégions ici la discussion des problèmes techniques et méthodologiques liés à l'utilisation d'un tel test. Les exemples présentés sont choisis dans ce but, en préalable indispensable à l'analyse des résultats d'expériences de choix entre variétés végétales.

Les fourmis champignonnistes (Attini) des genres Atta et Acromyrmex sont d'importants ravageurs des plantes cultivées ou spontanées du monde tropical américain (CherretT, 1986 ; Fowler, 1986). Ces fourmis cultivent un basidiomycète symbiotique sur un substrat végétal frais constitué de fleurs et de feuilles découpées dans l'environnement du nid. Ce symbiote est l'unique source de nourriture des larves ; les adultes ingèrent également de la sève issue des plantes découpées (LITTLEDYKE \& CHERRETT, 1976 ; Febvay \& Kermarrec, 1981 $a, b$; Febvay et al., 1984). Au vu de l'activité de découpe des végétaux, ces fourmis peuvent être considérées comme «polyphages » au sens écologique du terme (CHERRETT, 1972) ; en effet une très grande variété de plantes est exploitée (WEBER, 1966 ; CHERRETT, 1968). Face à cette large gamme de matériel végétal, les attines présentent cependant des préférences (AMANTE, 1967; CHERRETT, 1968 ; ROCKWOOD, 1976 ; FOWLER \& ROBINSON, 1977 ; POLLARD et al., 1983 ; PINTERA, 1983). A l'intérieur d'un même genre de plantes, certaines espèces sont résistantes aux attaques alors que des taxons proches s'avèrent particulièrement sensibles. Les différents mécanismes classiques de défense des plantes paraissent efficients. Dernièrement, les composés chimiques susceptibles d'être à l'origine du choix des ouvrières ont été particulièrement étudiés, soit dans le but de trouver de nouvelles substances pour lutter contre ces fourmis (répulsifs, antifongiques ; HubBell et al., 1983 ; HUBBell \& WieMER, 1983 ; CHEN \& WIEMER, 1984), soit en vue d'une sélection de variétés résistantes (FEBVAY et al., 1985).

Dans tous ces cas, les tests éthologiques utilisent 2 séries (ou plus) de confettis de limbe ou de confettis de papier filtre imbibés d'extraits à éprouver, déposés au hasard sur une grille. Le comportement d'affouragement des attines est stéréotypé : le choix des ouvrières s'opère sur la plateforme de récolte et un confetti prélevé est, dans plus de 99 p. 100 des cas, transporté à l'intérieur du nid. L'analyse de ce comportement par enregistrement vidéo a été réalisé par HUBBELL \& WIEMER (1983), mais il nécessite l'exploitation a posteriori de la bande enregistrée.

Sur la base de ce principe nous avons réalisé l'auto- matisation complète des tests de préférence à l'aide d'un micro-ordinateur couplé à une caméra.

\section{MATÉRIEL ET DISPOSITIF EXPÉRIMENTAL}

\section{A. Matériel informatique et de prise de vue}

Le micro-ordinateur utilisé est un Apple $\mathrm{II}+{ }^{\mathbb{B}}$ (Apple Computer, Inc.) avec 48000 octets de mémoire vive. Il est équipé de 2 lecteurs de disquettes souples et d'un moniteur noir et blanc pour les sorties vidéo. Le $2^{\mathrm{e}}$ lecteur facilite le stockage des fichiers résultats sur une disquette réservée à cet usage. L'impression de résultats ou de graphiques est réalisée par une imprimante matricielle standard. Le microordinateur a été équipé d'une carte horloge Thunderclock Plus ${ }^{\mathbb{R}}$ (Thunderware, Inc.) qui jouera le rôle de chronomètre pendant l'expérience. Les images enregistrées par la caméra sont digitalisées par la carte Dithertizer $\mathrm{II}^{\mathbb{B}}$ (Computer Stations, Inc.; vitesse de balayage de $1 / 60 \mathrm{~s}$; résolution de 53760 pixels). Le contraste et la brillance de l'image visualisée sur le moniteur peuvent être réglés par le manipulateur à l'aide de 2 «manettes de jeu ». La caméra utilisée doit permettre une synchronisation extérieure : c'est en effet l'ordinateur, par l'intermédiaire de la carte de digitalisation, qui commande les balayages de la caméra. En dehors de cette caractéristique, toute caméra de magnétoscope peut être utilisée. Nous avons opté pour une caméra HV-17A ${ }^{(\mathbb{R})}$ (Hitachi Denshi, Ltd.) équipée d'un objectif zoom $28 / 85 \mathrm{~mm}$, qui permet d'ajuster l'image de la zone expérimentale au format optimal.

\section{B. Plateforme de test}

La plateforme du test est une réplique légèrement modifiée de celle décrite par HUBBELL \& WIEMER (1983) (fig. 1). La partie supérieure d'un caisson en plexiglass est reliée par plusieurs ponts en verre au nid à tester. La plateforme de ce caisson reçoit une plaque en plexiglass gris opaque portant une grille de $25 \times 25$ carrés élémentaires de $2 \mathrm{~cm}$ de côté. Le centre de chaque carré est percé d'un trou de $1,5 \mathrm{~mm}$. Cette plateforme de test repose sur une " boîte à lumière " composée de 3 tubes au néon recouverts d'un verre diffusant. Grâce au préréglage du contraste (ouverture du diaphragme de l'objectif et réglage avec les manettes de jeu), l'image envoyée à l'ordinateur et visible sur le moniteur est composée de petites taches blanches dues à la lumière passant par les trous de la plaque. Le dépôt des confettis obture ces trous et l'image-écran est totalement noire. Le principe du test est le suivi au cours du temps de la prise des confetti, par le rythme d'apparition des taches lumineuses.

\section{Matériel animal}

Les expériences sont conduites sur des nids provenant de l'île de la Grande-Terre (Est-Guadeloupe) et conservés en activité au laboratoire à $25-30^{\circ} \mathrm{C}, 70$ 80 p. $100 \mathrm{HR}$. Ils contiennent tous une reine féconde et la meule fongique a un volume d'environ 0,5 à $1 \mathrm{dm}^{3}$. En dehors des périodes d'expériences, ces nids sont 


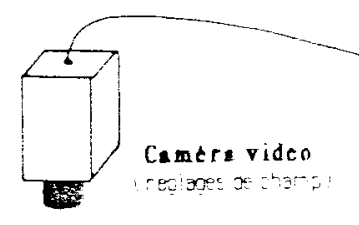

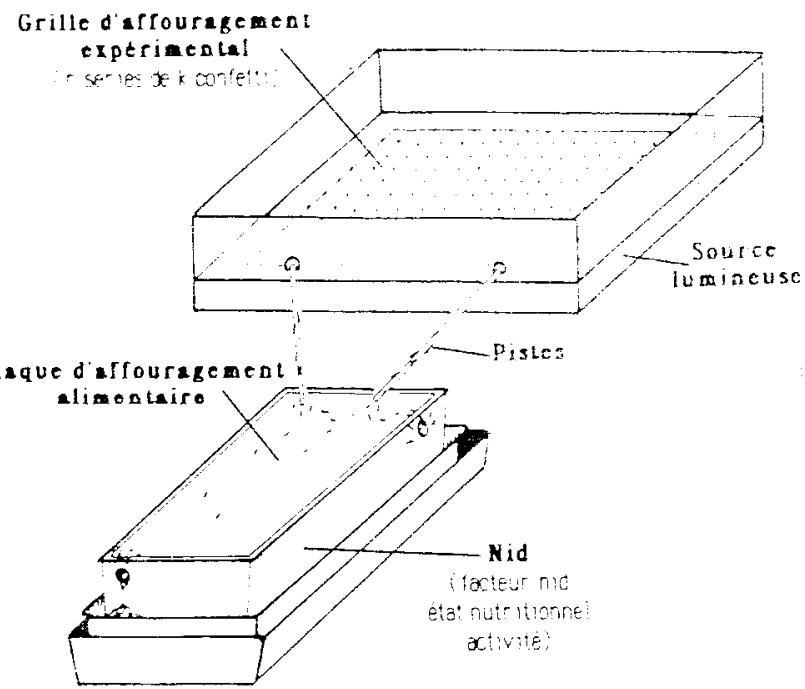

Figure 1

Dispositif expérimental géré par le logiciel « Attinetho » : enregistement automatisé de l'affouragement sur $n$ séries de confettis végétaux ou de papier filtre imbibés; $n=2$ à 7 .

approvisionnés quotidiennement avec du matériel végétal frais, varié et apprécié.

\section{LOGICIEL}

Le logiciel intitulé «Attinetho V2.0 Juin 1985 »a été réalisé par les auteurs. Les parties du programme en conversationnel ainsi que les routines de contrôle sont écrites en Basic Applesoft de grande souplesse et de maintenance facile. Les routines d'analyse d'images écrites en langage assembleur du microprocesseur 6502 autorisent des vitesses d'analyse importantes. Ce logiciel comporte les 3 parties indépendantes suivantes :

\section{$1^{\text {re }}$ partie : Définition de l'environnement expérimental}

Le programme a été réalisé avec le souci d'être utilisable dans des conditions expérimentales les plus variées possibles. Lors des essais de choix, l'expérimentateur peut être amené à éprouver en parallèle l'attractivité d'un nombre quelconque de séries de confettis (végétal ou papier filtre imbibé d'extraits). Aussi le nombre de cases par série et leur repérage au hasard sur la grille peuvent changer d'une expérience à l'autre; il sera fonction entre autres, du nombre de séries testées. La taille de la grille d'expérience est également adaptable. Si pour tester en parallèle 2 séries de confettis une grille de $12 \times 12$ carrés (soit 72 confettis de chaque série) est suffisante, il faudra une grille de $20 \times 20$ pour tester 5 séries différentes de 80 confettis.

Aussi, la $1^{\text {re }}$ partie du logiciel permet d'attribuer à chaque case d'une grille (de dimension déterminée et pour un nombre de séries défini) le numéro de série
Experimental device for automatic recording of foraging on $n$ series of leaf or paper discs; $n=2$ to 7 .

du confetti à déposer. Ces caractéristiques sont stockées sur disquette dans un fichier qui sera lu en début d'expérimentation (cf. $2^{\mathrm{e}}$ partie du logiciel). L'attribution d'un numéro de série pour chaque case peut être faite au hasard par l'ordinateur (cas le plus général), mais l'expérimentateur peut définir lui-même la géographie de sa grille (tests sur la prise des confettis en fonction de leur position sur le plan, ...). Le fichier réalisé pour une expérimentation donnée est bien sûr réutilisable. La géométrie de la grille définie par ce fichier peut être visualisée sur l'imprimante et le sera obligatoirement lors du dépôt des confettis.

\section{$2^{\mathrm{e}}$ partie : Déroulement d'une expérience}

Le programme de suivi d'une expérience comporte 7 étapes séquentielles. Les $1^{\text {res }}$ étapes précèdent le dépôt des confettis sur la grille d'expérience éclairée.

$I^{r e}$ étape : lecture du fichier

Définissant l'environnement expérimental de l'expérience (cf. ci-avant).

\section{$2^{e}$ étape : réglage de l'image}

En modifiant la focale du téléobjectif équipant la caméra, l'ouverture du diaphragme et en réglant les manettes de jeu de l'ordinateur, l'opérateur met au point une image, visualisée sur le moniteur, de taille et de contraste optimaux. Les positions relatives caméra/plateforme de choix ainsi que les réglages ne doivent alors plus être modifiés.

\section{$3^{e}$ et $4^{e}$ étapes}

L'expérimentateur n'intervient pas, la recherche des points lumineux et la mise en mémoire des coordonnées de leur centre sont automatiques. Un tri de ces 
points les relie ensuite aux cases initialement définies par le fichier de l'environnement expérimental. Cette étape permet de référencer les confettis de chaque case à la série à laquelle chacun appartient.

\section{Se étape}

Afin de s'assurer que les points mis en mémoire correspondent effectivement aux trous de la grille expérimentale, l'expérimentateur peut effectuer une série de balayages (255 au maximum). Pour tester ces prises de vue, l'ordinateur vérifie que les points mémorisés restent bien éclairés. L'expérimentateur est informé du nombre d'erreurs rencontrées ; si celui-ci n'est pas nul, il a alors la possibilité d'améliorer les réglages (étape $\mathrm{n}^{\circ} 2$ ).

\section{6e étape}

L'expérimentateur procède au dépôt des confettis et une $1^{\text {re }}$ prise d'image automatique vérifie si l'occultation des trous est bien réalisée. Dans le cas contraire, l'expérimentateur en est averti et, par visualisation de l'image sur le moniteur, peut aisément ajuster les dépôts défectueux.

\section{7e étape}

Cette dernière étape est le suivi proprement dit de la prise des confettis par les fourmis. Les ponts d'accès des fourmis, du nid à la plateforme de test, sont mis en place. Au fur et à mesure du déroulement de l'expérience, le moniteur affiche le temps écoulé, le nombre de confettis prélevés ainsi que, pour chaque série, le pourcentage de confettis prélevés par rapport aux déposés. Au début de cette étape, une alternative est proposée :

- suivi de l'expérience jusqu'à la prise de tous les confettis,

- arrêt de l'expérience dès que le pourcentage de confettis prélevés dans une série atteint une valeur fixée.

Toutefois, la possibilité est conservée d'interrompre à tout moment le suivi de l'expérimentation par la touche sortie (ESCape).

Une fois cette étape commencée, la présence de l'expérimentateur n'est plus requise. En fin de manipulation, les résultats seront automatiquement sauvegardés sur disquette. La durée de chacune des étapes décrites ci-dessus est reportée au tableau 1.

\section{$3^{\text {e }}$ partie : Exploitation des résultats}

Un des avantages supplémentaires et appréciables de l'utilisation d'un micro-ordinateur est l'exploitation immédiate des données enregistrées par des programmes appropriés. Le fichier résultat obtenu à la fin de l'expérimentation est à accès séquentiel : il mémorise chronologiquement pour chaque confetti son numéro de série ainsi que le temps au bout duquel il a été prélevé. Ce fichier est par conséquent aisément exploitable et la $3^{\mathrm{e}}$ partie du logiciel Attinetho propose différentes options (non limitées) pour une exploitation standard des données :

- impression et manipulation des fichiers résultats,

- conversion des fichiers résultats en fichiers compatibles Visitrend/Visiplot ${ }^{(\mathbb{B})}$ (Personal Software) pour une analyse statistique de séries chronologiques et leur représentation graphique,

- test du khi2,

- test de comparaison de pentes.

\section{RÉSULTATS ET LIMITES DE LA MÉTHODE}

A titre d'exemple, nous présentons les résultats obtenus avec $A$. octospinosus au cours de 2 expérimentations types. La $1^{\text {re }}$ porte sur le comportement de choix vis-à-vis de 3 espèces végétales, l'igname adon et l'igname jaune (Dioscorea bulbifera L. et Dioscorea cayenensis cayenensis Lam., Dioscoréacées), ainsi que l'hibiscus ornemental (Hibiscus rosa-sinensis L., Malvacée). Trois séries de 75 confettis de feuilles de ces espèces sont déposées sur une grille de $15 \times 15$ cases (diamètre des confettis : $6 \mathrm{~mm}$ ). Le suivi de l'expérimentation est réalisé à l'aide du logiciel Attinetho avec arrêt de l'enregistrement des résultats dès que 50 p. 100 des confettis d'une série sont prélevés (pour

\section{TABLEAU 1}

Durée de chacune des étapes détaillées dans le texte, mesurées pour une expérimentation avec 144 confettis (grille de $12 \times 12$ ) et avec 400 confettis (grille de $20 \times 20)$.

Duration of the experimental steps described in the text, calculated for assays with $144(12 \times 12$ grid $)$ and 400 discs $(20 \times 20$ grid $)$.

\begin{tabular}{|c|c|c|c|}
\hline Etapes du logiciel & $\begin{array}{l}\text { Grille de } \\
12 \times 12 \text { cases }\end{array}$ & $\begin{array}{c}\text { Grille de } \\
20 \times 20 \text { cases }\end{array}$ & Observations \\
\hline $\begin{array}{l}\text { 1re partie : } \\
\text { Création du fichier « environnement } » \ldots \ldots \ldots .\end{array}$ & $1 \mathrm{mn}$ & $7 \mathrm{mn}$ & $\begin{array}{l}\text { Pour un type d'expérimentation, cette } \\
\text { étape n'est effectuée qu'une fois }\end{array}$ \\
\hline \multicolumn{4}{|l|}{ 2e partie : } \\
\hline 1. Lecture du fichier. & \multirow{2}{*}{\multicolumn{2}{|c|}{$\begin{array}{l}4 \mathrm{~s} \\
\text { de } 1 \mathrm{mn} \text { à } 5 \mathrm{mn}\end{array}$}} & \multirow{8}{*}{$\begin{array}{l}\text { Selon l'habitude de l'expérimentateur } \\
\text { l'expérimentateur peut déposer les con- } \\
\text { fettis sur une plaque en verre, en dehors } \\
\text { du champ expérimental, au cours de } \\
\text { ces étapes pendant lesquelles il n'inter- } \\
\text { vient pas }\end{array}$} \\
\hline 2. Réglage de l'image $\ldots \ldots \ldots \ldots$. & & & \\
\hline 3. Détermination des coordonnées. & $6 \mathrm{mn} 15 \mathrm{~s}$ & $18 \mathrm{mn}$ & \\
\hline 4. Tri des points enregistrés $\ldots \ldots \ldots$ & $1 \mathrm{mn} 15 \mathrm{~s}$ & $2 \mathrm{mn} 50 \mathrm{~s}$ & \\
\hline 5. Vérification sur 100 balayages $\ldots \ldots \ldots \ldots$. & $30 \mathrm{~s}$ & $1 \mathrm{mn}$ & \\
\hline 6. Dépôt des confettis ................... & \multirow{2}{*}{\multicolumn{2}{|c|}{$\begin{array}{l}\text { étape non automatisée } \\
\text { inférieur à } 1 \mathrm{~s}\end{array}$}} & \\
\hline 7. Vérification de l'occultation .............. & & & \\
\hline 8. Expérimentation, analyse d'images $\ldots \ldots \ldots$. & 12 images en 10 & images en $10 \mathrm{~s}$ & \\
\hline
\end{tabular}

Total de préparation : de 20 à $60 \mathrm{mn}$ selon la taille de la grille, le nombre de séries et la nature des confettis (végétal, papier filtre imbibé...) 
Fichier : RE-18/7-18H

Titre de l'experiatentation : TEST-26 H/A/lJ (3)

Nonbre de series : 3

Nonbre de confetti deposes par serie: 75

NOHS DES SERIES :

Serie No 1 : HIBISCUS

Seris No 2 : ADON

Serie No 3 : IGNAHE JaLRE

Chronologie de la prise de confettl:

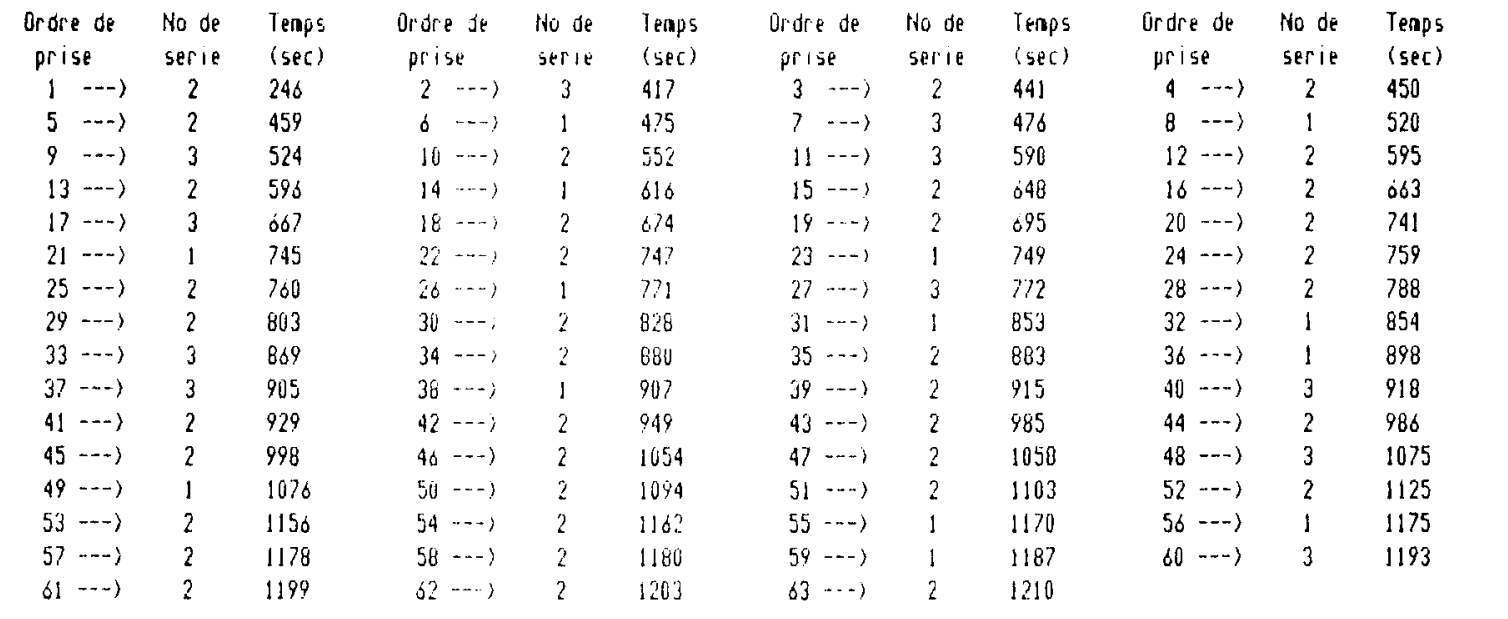

Figure 2

Sortie imprimée du fichier résultat. Il contient chronologiquement le temps de prélèvement d'un confetti et son numéro de série.

des expériences de choix, cette limite est raisonnable; au-delà, le choix des fourmis peut être biaisé par la disparition des confettis de la série la plus appétante). L'expérimentation a duré $1210 \mathrm{~s}(20 \mathrm{mn} 10 \mathrm{~s})$, 63 confettis ont été prélevés au total (38 de la série igname la plus appétante, 14 pour l'hibiscus et 11 pour la moins appréciée). Le fichier résultat (fig. 2) permet la réalisation immédiate de la figure 3 avec le logiciel graphique. Différentes analyses statistiques

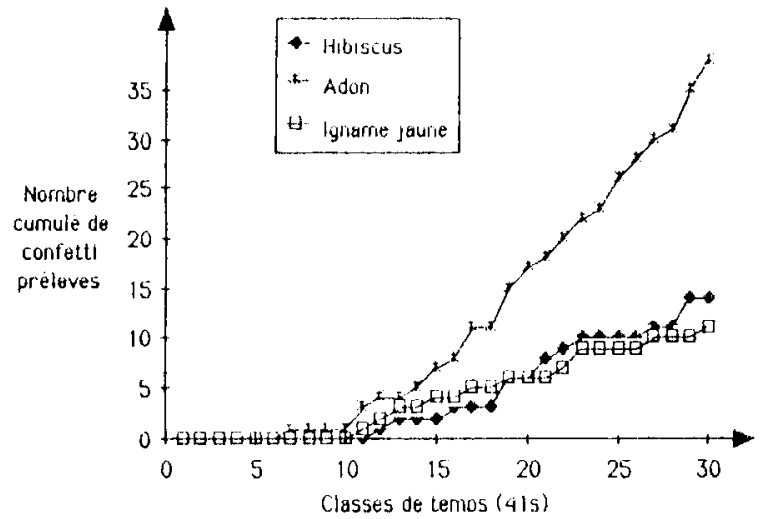

Figure 3

Exemple de visualisation graphique directement réalisable à partir du fichier résultat. Elle représente ici, pour 3 séries de confettis végétaux (igname adon, igname jaune et hibiscus), l'évolution du nombre cumulé de confettis prélevés en fonction du temps.

Example showing the quickly drawn graphical version of a result file : cumulative number of pick-up discs with time, for 3 series of leaf discs (adon and yellow yam and hibiscus).
Printed output of the result file, containing chronologically the time of pick-up and the serial number of each disc.

(khi2, comparaison de pentes...) peuvent également être directement demandées.

Un $2^{\mathrm{e}}$ type de résultats concerne l'intensité d'affouragement sur des confettis de papier filtre imbibés par des solutions de concentrations différentes en certains produits chimiques. La figure $4 a$ illustre un modèle de réponse différentielle à une gamme de concentrations croissantes d'un stimulant d'affouragement pour $A$. octospinosus (Tang ${ }^{(B)}$ : solution sucrée contenant des huiles essentielles d'orange et des arômes artificiels).

Dans les 2 cas présentés, le coefficient de régression linéaire des courbes enregistrées constitue une mesure de l'appétence des fourmis pour les confettis de chaque série (vitesse moyenne d'affouragement). Pour pallier les variations dues à des différences d'activité des nids ou à leurs facultés intrinsèques de discrimination, ces pentes peuvent être rapportées à des valeurs témoins (pentes maximale et minimale...) pour former des indices plus fiables. La figure $4 \mathrm{~b}$ montre la définition et la variation d'un tel indice dans le cas de notre $2^{\mathrm{e}}$ expérience.

Nous commentons dans ce qui suit certaines limites liées à l'utilisation de notre dispositif et essayerons d'en dégager en conclusion les principaux avantages et les champs d'application potentiels.

La conception du logiciel Attinetho a été menée avec le souci permanent de le rendre utilisable dans les conditions expérimentales les plus variées possibles par un expérimentateur sans connaissances particulières en informatique. Ce dernier est sollicité en conver- 

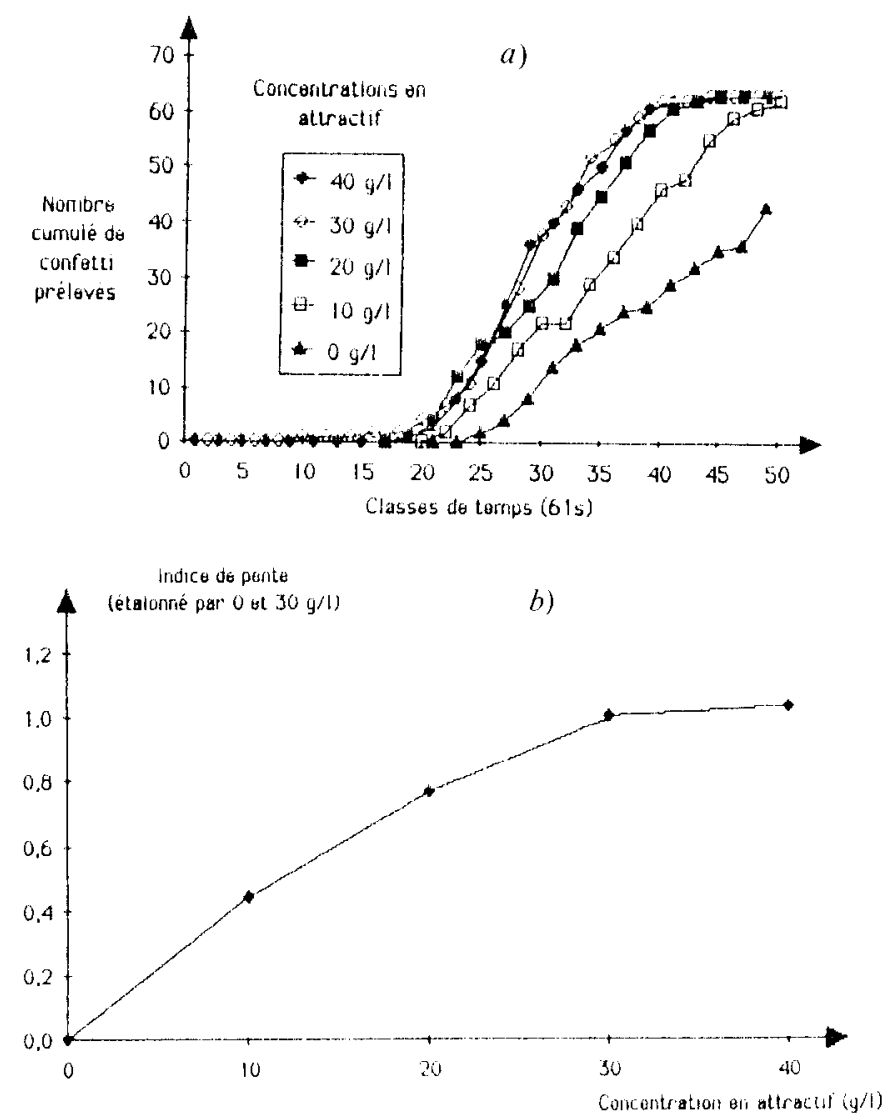

Figure 4

Résultats d'une expérience d'affouragement sur des confettis de papier filtre imbibés d'un stimulant d'affouragement. a) courbes de prélèvements des confettis en fonction du temps pour des concentrations croissantes en stimulant ; b) évolution de l'indice de pente en fonction de la conceniration en stimulant.

$$
\text { Indice utilisé : } I_{H . B}(t)=\frac{P_{1}-P_{B}}{P_{H}-P_{B}}
$$

Results of a foraging experiment with paper discs soaked in a pickup stimulant : a) curves of disc collection versus time, for increasing concentrations of stimulant ; b) slope index as function of stimulant concentration.

$$
\text { Index used : } I_{H . B}(t)=\frac{P_{t}-P_{B}}{P_{H}-P_{B}} \text {. }
$$

sationnel et informé de toute erreur, avec la possibilité d'y remédier.

Les limites techniques de cette automatisation découlent de différents facteurs :

- Parasites lumineux et électriques : La liaison caméra/micro-ordinateur est particulièrement sensible aux interférences parasites des champs électromagnétiques de l'environnement. Lors des premiers essais, ces champs parasites ont en effet entraîné l'enregistrement d'une proportion non négligeable de prises de confettis excédentaires. La dernière version Attinetho (V2.O Juin 1985) réalise 2 prises d'images successives afin de vérifier la réalité du prélèvement; les parasites étant aléatoires, leur influence est ainsi éliminée. De plus, l'utilisation des plus grandes grilles $(21 \times 21$ ou $25 \times 25$ ) nécessite une relative obscurité ambiante pour réduire l'effet d'interférences optiques (reflets et diffusion) sur l'optimisation du contraste.

- Limite liée à la place mémoire disponible: Avec un Apple II + de 48000 octets de mémoire vive, une expérimentation avec une grille de $25 \times 25$ cases (soit 625 confettis) peut être conduite sans problème. Pour une réponse statistique correcte, 60 confettis par série déposée sont suffisants. Une grille de $21 \times 21$ cases permet ainsi de tester 7 séries (de 63 confettis chacune) en parallèle.

- Surestimation du nombre de confettis prélevés: Un confetti pris par une fourmi puis abandonnẻ sera évidemment enregistré comme prélevé. Avec l'espèce étudiée, ce cas n'est observé qu'à une fréquence toujours inférieure à 1 p. 100 du nombre total de confettis prélevés. Les déplacements accidentels de confettis par le passage des fourmis sont par contre moins rares et nous ont conduits à utiliser une grille comportant des cases de $2 \mathrm{~cm}$ de côté. Pratiquement, le risque est fortement diminué par un rapport taille des ouvrières affourageuses/distance entre trous supérieur à 1,5. Dans ces conditions et pour les expérimentations limitées à 50 p. 100 de prise des confettis d'une série, le pourcentage de confettis déplacés reste toujours inférieur à $5 \mathrm{p}$. 100 et se situe généralement aux environs de 1 à 2 p. 100. Le niveau de cette erreur reste admissible, d'autant plus que les confettis déplacés le sont au hasard. Ce "bruit ", même s'il influe sur la puissance des tests statistiques, n'intervient en rien sur leurs conclusions.

Les limites biologiques apparues à l'utilisation concernent presque exclusivement la variabilité des réponses en fonction des conditions expérimentales et son influence sur la signification statistique des indices calculés. Le test du khi2 (comme le test binomial utilisé par HubBell \& WIEMER, 1983) n'utilise qu'une faible partie de l'information disponible (prises enregistrées à un temps donné et en conformité à l'hypothèse d'égalité des séries). Son utilisation est satisfaisante lors de réponses marquées, mais son relatif manque de puissance en limite l'intérêt dans certains cas (courbes dose-réponse par exemple). A l'inverse, le test de comparaison de pentes peut se révéler trop sensible, traduisant une inadéquation à ses hypothèses sous-jacentes dans certaines conditions expérimentales. Quoi qu'il en soit, le contrôle total du déroulement des prises permet d'adapter le meilleur traitement à chaque type d'expérience, ce qui n'est pas forcément le cas lorsque l'information recueillie est partielle.

L'origine des variations peut être décomposée en plusieurs facteurs plus ou moins indépendants. L'activité du nid, liée à sa taille, à son état nutritionnel ou à son rythme biologique endogène, intervient de façon prépondérante dans la réponse expérimentale. Son pouvoir discriminant vis-à-vis du substrat de récolte peut aussi être affecté par son état trophique (jeûne prolongé...). L'utilisation de l'indice défini en figure $4 \mathrm{~b}$ permet souvent de minimiser ces facteurs : " étalonnage 》 de l'activité du nid par la pente maximale observée et de l'étendue des réponses par un couple de pentes témoins. Plus gênante est, dans certains cas, la manifestation d'une variabilité des " goûts " intrinsèques des nids ; elle n'est d'ailleurs pas forcément fixe dans le temps; elle résulte vraisemblablement de l'« histoire individuelle » de ces nids et d'éventuels phénomènes d'apprentissage à court ou moyen termes. La neutralisation de ces variations passe par une définition assez rigoureuse des condi- 
tions expérimentales. Il est à noter que ces aléas ne dépendent pas de notre dispositif expérimental mais du caractère complexe des sociétés de fourmis. Inversement, des adaptations aisées de ce dispositif permettraient justement d'étudier l'incidence et l'importance des facteurs cités sur le comportement d'affouragement des attines.

\section{CONCLUSION}

Si ce dispositif comporte des limites évidentes, il apporte aussi des avantages certains, dont nous laissons juge l'utilisateur des tests éthologiques de ce type : l'automatisation libère partiellement l'expérimentateur et nous avons déjà signalé l'intérêt du contrôle intégral du déroulement; l'avantage majeur nous semble cependant la réduction du temps d'expérience accompagné de la disponibilité immédiate des résultats, permettant de multiplier les répétitions et d'améliorer d'autant la fiabilité des conclusions.

Les domaines d'application sont relativement vastes et couvrent par exemple dans notre cas des tests de choix appliqués à la sélection variétale, des analyses du comportement d'affouragement (ou éventuellement de transport de couvain) ainsi que des tests d'activité biologique au cours du fractionnement chimique du matériel végétal étudié.

Le programme Attinetho a été créé et éprouvé sur une seule espèce de fourmi attine, mais il n'en est pas moins aisément adaptable à tout autre fourmi, insecte ou petit animal au comportement similaire, consistant grossièrement en un ramassage d'objets immobiles. A cet effet, le logiciel, accompagné d'une notice détaillée, peut être demandé directement aux auteurs.

Reçu le 20 novembre 1985. Accepté le 23 avril 1986.

\section{REMERCIEMENTS}

Ces travaux ont été financés par la CORDET (contrats Agronomie $\mathrm{n}^{\circ} \mathrm{C} 101,1983$ et $\left.\mathrm{C} 402,1984\right)$. Nous tenons à remercier J. TAYEB (I.N.R.A., Nantes) pour ses conseils sur les automatisations et son expérience en programmation ainsi que P. DURIS (I.U.T., Tours), dernière personne obligée de suivre visuellement les expériences afin de contrôler l'automatisation!

\section{RÉFÉRENCES BIBLIOGRAPHIQUES}

Amante E., 1967. A formiga sauva Atta capiguara praga das pastagens. Biologico, 33, 113-120.

Chen T. K., Wiemer D. F., 1984. A volatile leaf-cutter and repellent from Astronium graveolens. Naturwissenschaften, 71, 97-98.

Cherrett J. M., 1968. The foraging behaviour of Atta caphalotes L. (Hymenoptera, Formicidae). I : Foraging pattern and plant species attacked in tropical rain forest. J. anim. Ecol., 37, 387-403.

Cherrett J. M., 1972. Some factors involved in the selection of vegetal substrate by Atta cephalotes (L.) (Hymenoptera ; Formicidae) in tropical rain forest. J. anim. Ecol., 4l, 467-660.

Cherrett J. M., 1986. History of the leaf-cutting ant problem. Proc. Res. Conf. on Fire ants and Leaf-cutting ants, Gainesville (souspresse).

Febvay G., Kermarrec A., 1981a. Morphologie et fonctionnement $\mathrm{du}$ filtre infrabuccal chez une attine Acromyrmex octospinosus Reich (Hymenoptera; Formicidae) : rôle de la poche infrabuccale. Int. J. Insect Morphol. Embryol., 10, 441-449.

Febvay G., Kermarrec A., 1981b. Activités enzymatiques des glandes salivaires et de l'intestin moyen d'une fourmi attine (adultes et larves): Acromyrmex octospinosus Reich (Formicidae; Attini). Arch. Biol., 92, 299-316.

Febvay G., Decharme M., Kermarrec A., 1984. Digestion of chitin by the labial glands of Acromyrmex octospinosus Reich (Hymenoptera ; Formicidae). Can. J. Zool., 62, 229-234.

Febvay G., Bourgeois P., Kermarrec A., 1985. Antiappétants pour la fourmi attine, Acromyrmex octospinosus (Reich) (Hymenoptera; Formicidae) chez certaines espèces d'ignames (Dioscoreaceae) cultivées aux Antilles. Agronomie, 5, 439-444.

Fowler H. G., 1986. Economics of grass-cutting ants. Proc. Res. Conf. on Fire ants and Leaf-cutting ants, Gainesville (sous-presse).
Fowler H. G., Robinson S. W., 1977. Foraging and grass selection by the grass-cutting ant Acromyrmex landolti fracticornis (Forel) (Hymenoptera ; Formicidae) in habitats of introduced forage grasses in Paraguay. Bull. entomol. Res., 67, 659-666.

Hoy J. B., Globus P. A., Norman K. D., 1983. Electronic tracking and recording system for behavioral observations, with application to toxicology and pheromone assay. J. econ. Entomol., 76, 678680 .

Hubbell S. P., Wiemer D. F., 1983. Host plant selection by an attine ant, 133-154. In P. Jaisson: "Social insects in the tropics", II, Univ. Paris Nord, $252 \mathrm{p}$.

Hubbell S. P., Wiemer D. F., Adejare A., 1983. An antifungal terpenoid defends a neotropical tree (Hymenaea) against attack by fungus-growing ants (Atta). Oecologia, 60, 321-327.

Littledyke M., Cherrett J. M., 1976. Direct ingestion of plant sap from cut leaves by the leaf-cutting ants Atta cephalotes (L.) and Acromyrmex octospinosus (Reich) (Formicidae, Attini). Bull. entomol. Res., 66, 205-217.

Pintera A., 1983. Selection of plants utilized by Atta insularis in Cuba (Hymenoptera ; Formicidae). Acta entomol. Bohemosto., 80, 13-20.

Pollard G. V., Riley R., Wattie E., 1983. Preliminary investigations on the selection of citrus species by the leaf-cutting ant, Acromyrmex octospinosus (Reich) (Formicidae, Attini). Trop. Agric. (Trinidad), 60, 282-285.

Rockwood L. L., 1976. Plant selection and foraging patterns in two species of leaf-cutting ants (Atta). Ecology, 57, 48-61.

Weber N. A., 1966. Fungus-growing ants. Science N. Y., 153, 587604. 